\title{
Photoionization of xenon below the atomic ionization potential
}

\author{
P. Laporte \\ Equipe de Spectroscopie, Centre National de la Recherche Scientifique (LA 171), 158 bis cours Fauriel, \\ 42023 Saint Etienne, Cedex, France \\ V. Saile \\ HASYLAB, Deutsches Elektronen-Synchrotron (DESY), 2000 Hamburg 52, Federal Republic of Germany
}

\author{
R. Reininger, U. Asaf, and I. T. Steinberger \\ Racah Institute of Physics, The Hebrew University, Jerusalem, Israel \\ (Received 29 November 1982)
}

\begin{abstract}
Experiments using monochromated synchrotron radiation revealed that for densities of the order of $10^{19}$ atoms $/ \mathrm{cm}^{3}$ and more, xenon exhibits a continuous photoresponse excitation spectrum below the atomic ionization potential $(12.12 \mathrm{eV})$. The lower limit of the continuum is at about $11.10 \mathrm{eV}$, the energy difference between the ground state of the molecular ion $\mathrm{Xe}_{2}{ }^{+}$and that of the free atom. This is attributed to excimer formation at the wings of the atomic absorption lines followed by ionization and creation of xenon dimer ions. Dips appearing in the continuum very near to positions of atomic lines are discussed invoking the quasistatic theory.
\end{abstract}

It has been repeatedly pointed out that liquid xenon near the triple point has electronic properties similar to that of a crystalline photoconducting insulator: The electron mobility is very high ${ }^{1,2}$; there exists a rather sharp photoconduction threshold very near to the position of the threshold in the solid ${ }^{3}$ and excitonic peaks as in the solid are observed in the reflection spectrum of the liquid as well. ${ }^{4,5}$ In following the evolution of the exciton peaks with density, it is observed that the peaks are distinct from atomic lines broadened and shifted by molecular interactions. ${ }^{6,7}$ Thus xenon can serve as a convenient model system ${ }^{8,9}$ for the study of the evolution of energy bands of a nonmetal with the decrease of interatomic distance simply by recording the changes of the electronic properties upon gradual compression of the gas to densities nearing that of the solid.

The experiments were performed using the synchrotron radiation source in HASYLAB at the Deutsches Elektronen Synchrotron in Hamburg. The Honormi monochromator, cryostat, and data-acquisition system $^{10}$ was used. The stainless-steel sample cell was equipped with a

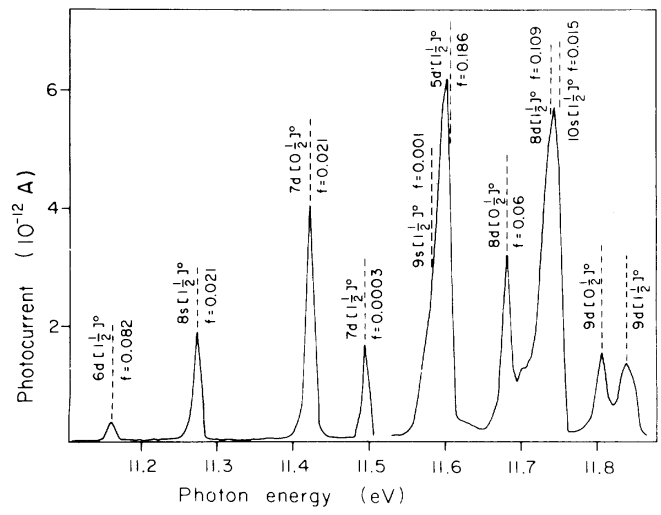

FIG. 1. Photocurrent as a function of photon energy for $\rho_{N}=10^{18}$ atoms $/ \mathrm{cm}^{3}$.
LiF front window: The inner side of the window was covered by a pair of intertwined gold electrodes prepared by sputtering. The interelectrode distance was $0.36 \mathrm{~mm}$; a potential difference of $2 \mathrm{~V}$ was applied for all experiments. Ultrahigh vacuum could be achieved in the cell; the cell could also withstand pressures of up to 100 atmospheres. Temperature variations from $77-400 \mathrm{~K}$ could also be obtained with the same construction. Deutsche l'Air Liquide N 47 grade xenon gas was used without further purification. The all-stainless-steel gas-handling system was baked under pumping for several hours before starting the experiments; the base pressure in the system and in the cell was of the order of $10^{-7}$ Torr. Before letting in the xenon gas, the system and the cell were repeatedly purged by helium at pressures of several atmospheres.

Figure 1 shows the observed photocurrent (without corrections or normalization) at a low density $\left(\sim 10^{18}\right.$ atoms $/ \mathrm{cm}^{3}$ ) as a function of the photon energy of the incident light. The current peaks coincide in position with the respective atomic lines, but they are considerably broader. The instrumental linewidth at half height was about $0.02 \mathrm{eV}$. No photoresponse was seen below the 11.160-eV peak. Above the highest energy peak shown, the LiF cutoff prevented further observations; in fact, even the two highest-energy peaks in Fig. 1 fall in the absorption-edge region of the window and therefore their shape and height could not be rendered correctly. It is of interest to compare these results with those obtained by Huffman and Katayama. ${ }^{11}$ These authors measured ionization currents at number densities much lower $\left(\leq 10^{16}\right.$ atoms $/ \mathrm{cm}^{3}$ ) than in our work. All lines observed by these authors and situated at photon energies $h v<11.85 \mathrm{eV}$ were seen in the course of the present work as well (Fig. 1), though somewhat broadened. The hierarchy of intensities is also similar for the two works. Because of the general broadening, the two neighboring lines associated with the $9 s\left[1 \frac{1}{2}\right]^{0}$ and $5 d^{\prime}\left[1 \frac{1}{2}\right]^{0}$ levels are not resolved in Fig. 1, though they are distinct in Ref. 11; the same is true about the two levels $8 d\left[1 \frac{1}{2}\right]^{0}$ and $10 s\left[1 \frac{1}{2}\right]^{0}$. The transitions to 


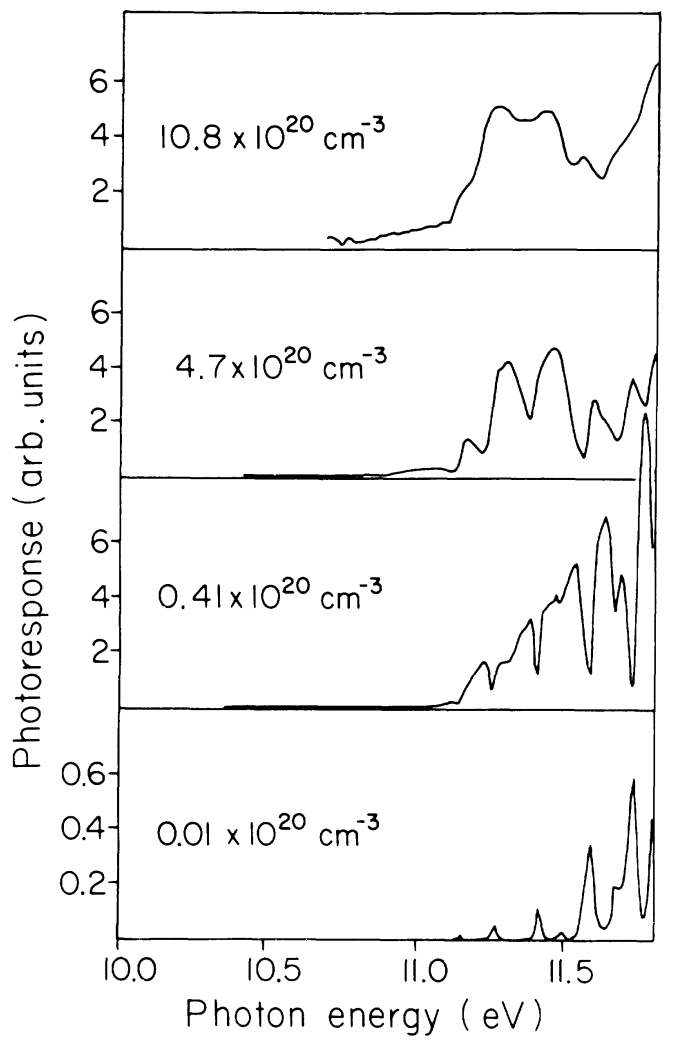

FIG. 2. Photocurrent normalized to an equal number of incident photons and divided by the zero-field mobility as a function of the photon energy at four densities. Spectra were taken at room temperature.

the levels $9 d\left[0 \frac{1}{2}\right]^{0}(11.801 \mathrm{eV})$ and $9 d\left[1 \frac{1}{2}\right]^{0}(11.835 \mathrm{eV})$ were not observed in Ref. 11, since the intensity of the argon continuum source employed by Huffman and Katayama was zero in this spectral region.

The use of a closed cell in the present work (see also Ref. 12) made it possible to study the influence of increasing density on the photoionization excitation spectrum. The photoresponse, as presented in Fig. 2, was obtained from the photoelectric current measured at $2 \mathrm{~V}$ by normalization to an equal number of incident photons, and division by the electron mobility. For the normalization, the relative number of photons passing through the $\mathrm{LiF}$ window was measured as a function of photon energy. Division by the zero-field electron mobility ${ }^{2}$ was necessary in order to separate the density dependence of the mobility from the density dependence of the carrier concentration. Figure 2 presents the photoresponse at four densities at room temperature, corresponding to a pressure range of about 50 mbar to 40 bar. The graphs were selected from an extensive series covering this density range in such a manner as to emphasize the qualitative changes. Note that for $h v>11.7 \mathrm{eV}$ the spectrum refers to a strongly absorbing region of the lithium fluoride window, thus the correction for the absorption is large and therefore no quantitative conclusions should be drawn from the graphs in this region.

When comparing the four graphs of Fig. 2 the following points stand out.

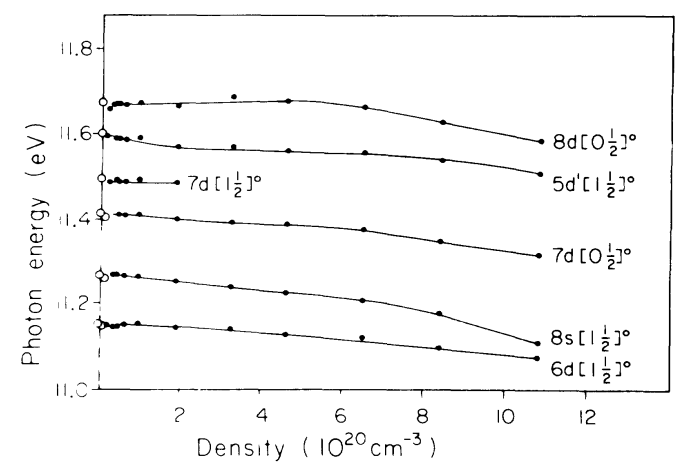

FIG. 3. Density dependence of the position of the peaks (open circles) and dips (solid circles) corresponding to six atomic transitions.

(a) The lowest-density graph exhibits peaks; in the next one presented, the peaks become dips on a slowly varying background. By increasing the density further, the dips shift to lower photon energies, broaden, and eventually wash out.

(b) The photoresponse for $\rho_{N} \sim 10^{18} \mathrm{~cm}^{-3}$ is smaller than that of the higher densities by an order of magnitude. However, for the other three densities not only the order of magnitude stays the same, but, if the minima are disregarded, the spectra can be fitted with envelopes of roughly similar shapes and heights (ignoring, as suggested above, the region $h v>11.7 \mathrm{eV}$ ).

(c) The envelopes of the three higher-density graphs drop sharply on the low-energy side. The position of this threshold shifts slightly with increasing density to lower photon energies: From $11.16 \mathrm{eV}$ for $\rho_{N}=0.41 \times 10^{20}$ atoms $/ \mathrm{cm}^{3}$ to $11.11 \mathrm{eV}$ at the highest density presented.

(d) For the three spectra corresponding to the higher densities there is a low-energy tail beyond the drop mentioned in (c). With increasing density this tail appears already at lower photon energies, and its contribution to the photoresponse at the position of the drop becomes more and more significant. At still higher densities, reported separately, ${ }^{13}$ this tail becomes the dominant feature of the spectrum.

Figure 3 shows the photon energies of the various peaks and the respective dips as a function of the number density. It is seen that the general trend is a slight shift to lower photon energies with increasing density. Simultaneously the dips become broader and shallower, as demonstrated in Fig. 2.

For the discussion of the mechanism underlying the reported effects, it is useful to note again that the envelope of the photoresponse continuum (above $\sim 11.1 \mathrm{eV}$ ) changes little with density in a wide range, though for the same density range, the low-energy tail changes markedly. At densities above those discussed here, ${ }^{13}$ this tail can be shown to be due to photoconductivity proper; namely, to direct excitations from a continuous ground state (valence band) into a continuous quasifree excited state (conduction band), the position of which is determined by the average interaction of thermal or nearly thermal electrons with the medium. Therefore one is naturally led to the assumption that, in contrast with the effects in the tail, most of the response at the densities in question is due to photoionization, and is governed by atomic or molecular interactions. 
It was seen in Fig. 1 that at the lowest densities, photoresponse appears at the positions of atomic absorption lines for photon energies well below the ionization energy $(12.12 \mathrm{eV})$ of the free xenon atom. As in Ref. 11, the response is attributed to the formation of xenon excimers that may relax vibrationally until crossing the potential curve of the dimer ion. Schematically

$$
\begin{aligned}
& \mathrm{Xe}+\mathrm{Xe}+h v \rightarrow \mathrm{Xe}_{2}^{*}, \\
& \mathrm{Xe}_{2}^{*} \rightarrow \mathrm{Xe}_{2}^{+}+e^{-} .
\end{aligned}
$$

The two xenon atoms in (1a) are nearest neighbors at the instant of the excitation by the photon. In principle, (1a) might also happen in two steps, starting with atomic excitation and followed by collision with an atom leading to excimer formation, as proposed by Hornbeck and Molnar. ${ }^{14}$ Direct ionization experiments of the van der Waals molecule $\mathrm{Xe}_{2}$ in a supersonic beam ${ }^{15,16}$ showed that (1b) is, indeed, a highly probable process that does not involve collision with a further Xe atom. For these processes the photon energy $h v$ must conform with the inequality

$$
I_{G}(\mathrm{Xe})-D\left(\mathrm{Xe}_{2}{ }^{+}\right) \leq h v \leq I_{G}(\mathrm{Xe}),
$$

where $I_{G}(\mathrm{Xe})$ represents the ionization energy of the xenon atom and $D\left(\mathrm{Xe}_{2}{ }^{+}\right)$the dissociation energy of the xenon molecular ion. The very small kinetic energies and the negligible energy of the vibrational ground state of $\mathrm{Xe}_{2}{ }^{+}$ were ignored in the above considerations. The threshold energy can be calculated as

$$
I_{G}(\mathrm{Xe})-D\left(\mathrm{Xe}_{2}{ }^{+}\right)=I_{G}\left(\mathrm{Xe}_{2}\right)-D\left(\mathrm{Xe}_{2}\right),
$$

where $I_{G}\left(\mathrm{Xe}_{2}\right)$ is the adiabatic ionization potential and $D\left(\mathrm{Xe}_{2}\right)$ the dissociation energy of the van der Waals molecule $\mathrm{Xe}_{2}$. Substituting numerical values from the work of Dehmer and Dehmer ${ }^{15}$ into Eq. (3) we obtain the threshold energy value of $11.10 \mathrm{eV}$. On the other hand, Fig. 2 shows that the photoresponse continuum sets in at $11.11-11.16 \mathrm{eV}$, depending on the density, in good agreement with the above threshold. This fact suggests that all photoresponse in the continuum, whether at the atomic lines or not, should be attributed to processes described in Eqs. (1a) and (1b). Such a generalization of the Hornbeck-Molnar process for a continuum of photon energies also follows from considering the theory relevant at the densities in question to the absorption-line wings, namely, the nearest-neighbor quasistatic approximation. The applicability of this approximation in the red wings of the lines is manifested by the quadratic dependence of the absorption coefficient on the gas density. ${ }^{17,18}$ According to this approximation, there is a one-to-one correspondence between the frequency $v$ at each point of the wing of an absorption line and the distance $r$ of the excited atom to its nearest neighbor, the exact functional relationship between $r$ and $v$ being given by the details of the configurational energy diagram. It is, nevertheless, clear that decreasing $v$ on the low-frequency side of the absorption line implies excitation of atoms with lower values of $r$ along the potential curve representing attraction between the excited atom and its nearest neighbor. One may assume that smaller $r$ implies less probability of the excimer to decay by channels other than the formation of the molecular ion and concurrent liberation of an electron. It follows that the red wing of an absorption line can make a considerable contribution to the processes described in Eq. (1). This fact, along with the above comparison of the energy thresholds, leads to the realization that the indirect photoionization response [like that described in Eq. (1) and by Hornbeck and Molnar ${ }^{14}$ ] at an atomic absorption line should manifest as a rather broad band, especially at higher densities. The overlap of a set of neighboring bands creates the continuous absorption seen in Fig. 2. This is the central result of this paper.

One of the most striking features of Fig. 2 is the appearance of inverted peaks at higher densities. At first sight it is tempting to attribute the observed inversion to the dependence of the lifetime of the liberated electrons on the penetration depth of the incident radiation. Accordingly, the penetration depth $d=1 / \alpha$ ( $\alpha$ being the absorption coefficient) at the line center $v_{0}$ of a self-broadened line is very small $(\sim 10 \mathrm{~nm}),{ }^{17}$ thus for the center the lifetime might considerably be shortened due to recombination at the $\mathrm{Xe}-\mathrm{LiF}$ interface or by energy-transfer effects. On the other hand, such surface effects would not shorten the lifetime for the wings where the penetration depth is larger. Thus at the wings the lifetime could be appreciably longer than at the center, resulting in an inversion of the line. In the density range involved, $d$ either stays constant at the line center or even decreases somewhat with density, while it increases with density in the wings. ${ }^{17}$ Even if it stays constant, decreasing the density simply would imply an overall narrowing of the photoresponse profile; thus, except for a narrowing, a similar response would be obtained at low densities as at high densities, in contrast with experiment. If, on the other hand, $d$ for excitation at the line center decreases with increasing density, the conflict with experiment is even sharper, since this would imply that low, rather than high, densities favor line inversion.

The most likely explanation for the line-shape inversion is based on the competition between electron liberation and dimer ion formation [Eq. (1b)], and all other decay channels. According to the above discussion, an increase of $v_{0}-v$ implies transition with a smaller nearest-neighbor distance $r$. Therefore the process of Eq. (1b) becomes more dominant the higher the pressure and the larger the value of $v_{0}-v$. In other words, an inversion of the line shape may occur with increasing density.

The fact that the dips corresponding to atomic lines become broader and shallower at high densities can also be understood on the basis of the variations of the profile of absorption line: With increasing density the absorptionline profiles broaden and flatten simultaneously and cause broadening of the photoresponse profile with simultaneous flattening of the dips. The red shift of the dips and of the onset with increasing density, are at least partly due to the usual effect of attractive potentials, including many-body effects, especially at higher densities. One of these effects is expressed by the polarization energy $P^{+}$of the hole left in the dense medium after removal of the electron. For the density range involved, $P^{+}$has been calculated ${ }^{13}$ to increase from zero to $0.04 \mathrm{eV}$. This is nearly equal to the observed shift (Fig. 2) of the long-wavelength limit of the spectra in Fig. 1 with density. It should also be noted that the sense of variation of the onset is in full accord with the expected enhancement of the photoresponse at the line wings with density. The shift of the dips is markedly 
larger than expected from the values of $P^{+}$alone.

The indirect ionization of xenon, resembling the Hornbeck-Molnar process, ${ }^{14}$ but observed for a continuum of photon energies in the present work, is an interesting, novel way of creating free electrons in a dense gas, and its possible influence should be taken into account under appropriate conditions, especially when studying the spectroscopy and dynamics of excimers.

\section{ACKNOWLEDGMENTS}

The authors wish to express their thanks to several institutions [Deutsches Elektronen-Synchrotron (DESY), Deutscher Akademischer Austauschdienst (DAAD), and the Israel Commission for Basic Research] and numerous individuals for their contributions towards the success of this work.
${ }^{1}$ L. S. Miller, S. Howe, and W. E. Spear, Phys. Rev. $\underline{166}, 871$ (1968).

2S. S. S. Huang and G. R. Freeman, J. Chem. Phys. 68(4), 1355 (1978).

${ }^{3}$ U. Asaf and I. T. Steinberger, Phys. Rev. B $\underline{10}, 4464$ (1974).

${ }^{4}$ D. Beaglehole, Phys. Rev. Lett. 15, 551 (1965).

${ }^{5}$ I. T. Steinberger and U. Asaf, Phys. Rev. B $\underline{8}, 914$ (1973).

${ }^{6}$ P. Laporte and I. T. Steinberger, Phys. Rev. A 15, 2538 (1977).

${ }^{7}$ P. Laporte, J. L. Subtil, U. Asaf, I. T. Steinberger, and S. Wind, Phys. Rev. Lett. 45, 2138 (1980).

${ }^{8}$ R. Reininger, U. Asaf, P. Laporte, and I. T. Steinberger, in Proceedings of the Seventh International Conference on Conduction and Breakdown in Dielectric Liquids, Berlin, 1981, edited by W. F. Schmidt (unpublished), p. 69; J. Electrostat. 12, 123 (1982).

${ }^{9}$ R. Reininger, U. Asaf, and I. T. Steinberger, Chem. Phys. Lett. 90, 287 (1982).

${ }^{10}$ V. Saile, P. Gürtler, E. E. Koch, A. Kozevnikov, M. Ski- bowski, and W. Steinmann, Appl. Opt. 15, 2559 (1976).

${ }^{11}$ R. E. Huffman and D. H. Katayama, J. Chem. Phys. 45, 138 (1966).

12J. A. R. Samson and R. B. Cairns, J. Opt. Soc. Am. $\underline{56}, 1140$ (1966).

${ }^{13}$ R. Reininger, U. Asaf, I. T. Steinberger, V. Saile, and P. Laporte, Phys. Rev. B (in press).

14J. A. Hornbeck and J. P. Molnar, Phys. Rev. $\underline{84}, 621$ (1951).

${ }^{15}$ P. M. Dehmer and J. L. Dehmer, J. Chem. Phys. 68(8), 3462 (1978).

${ }^{16}$ C. Y. Ng, D. J. Trevor, B. H. Mohan, and Y. T. Lee, J. Chem. Phys. 65, 4327 (1976).

17P. Laporte and H. Damany, J. Phys. (Paris) 40,9 (1979).

${ }^{18}$ R. Reininger, U. Asaf, I. T. Steinberger, P. Laporte, S. Bernstorff, and V. Saile, in Proceedings of the Seventh International Conference on Vacuum Ultraviolet Radiation Physics, Jerusalem, August, 1983, edited by A. Weinreb and A. Ron (Hilger, London, in press). 